\title{
Impaired Preference Conditioning after Anterior Temporal Lobe Resection in Humans
}

\author{
Ingrid S. Johnsrude, ${ }^{1}$ Adrian M. Owen, ${ }^{1}$ Norman M. White, ${ }^{2}$ W. Vivienne Zhao, ${ }^{2}$ and Veronique Bohbot ${ }^{3}$ \\ ${ }^{1}$ Medical Research Council Cognition and Brain Sciences Unit, Cambridge, CB2 2EF United Kingdom, ${ }^{2}$ Department \\ of Psychology, and 'BBrain Imaging Centre, Montreal Neurological Institute, McGill University, Montreal, Quebec H3A 2T5, \\ Canada
}

Research with animals suggests that structures within the amygdaloid nuclear complex (ANC) are critical for acquiring associations between rewarding events and neutral stimuli, a form of conditioning often manifested in a subsequent preference for those (conditioned) stimuli. In this study, we investigated the relationship between the ANC and preference learning in humans. Three abstract monochrome patterns were presented to each subject over 180 trials in the context of a counting task requiring working memory. One pattern was paired with food reward on $90 \%$ of the trials in which it was presented and with no food reward on the other $10 \%$ of trials. The other patterns were similarly reinforced, but at ratios of $50: 50 \%$ and 10:90\% with reward and nonreward, respectively. Subsequently, a group of 21 normal participants preferred the pattern paired most often with reward to that paired least often

An extensive body of research on animals has linked structures within the amygdaloid nuclear complex (ANC) to emotional associative learning. Much of this work has focussed on aversive phenomena such as fear conditioning (Gallagher and Chiba, 1996; Killcross et al., 1997; Rogan et al., 1997; Walker and Davis, 1997; Davis, 1998; LeDoux, 1998; Fanselow and LeDoux, 1999; Fendt and Fanselow, 1999). Studies of fear and aversive conditioning in humans have produced results consistent with the animal literature: patients with bilateral (Bechara et al., 1995) and even unilateral (LaBar et al., 1995) ANC lesions do not show normal fear conditioning responses. Functional neuroimaging studies also suggest a role for the ANC in fear conditioning in humans (Büchel et al., 1998; LaBar et al., 1998; Morris et al., 1998).

It has long been recognized in the animal literature that selective amygdala lesions can affect the ability of animals to associate stimuli with reward value (Weiskrantz, 1956; Jones and Mishkin, 1972; Speigler and Mishkin, 1981; Gaffan and Harrison, 1987; Cador et al., 1989; Robbins et al., 1989; Gallagher et al., 1990;

\footnotetext{
Received Oct. 21, 1999; revised Jan. 12, 2000; accepted Jan. 20, 2000.

This work was supported by the Medical Research Council of Canada through a Career Investigatorship awarded to B. A. Milner. We gratefully acknowledge her assistance. I.S.J. was funded by the Wellcome Trust during the preparation of this manuscript. This work was conducted in the Neuropsychology/Cognitive Neuroscience Unit, Montreal Neurological Institute, McGill University, while I.S.J. and A.M.O. were working there. We thank the participants, the neurosurgeons A. Olivier, W. Feindel, and R. Leblanc, and numerous colleagues for helpful discussions.

Correspondence should be addressed to Ingrid S. Johnsrude, Medical Research Council Cognition and Brain Sciences Unit, 15 Chaucer Road, Cambridge CB2 2EF, UK. E-mail: ingrid.johnsrude@mrc-cbu.cam.ac.uk.

Copyright (C) 2000 Society for Neuroscience $\quad 0270-6474 / 00 / 202649-08 \$ 15.00 / 0$
}

with reward, and they did not explicitly relate their preferences to the conditioning procedure, but instead attributed them to the characteristics of the patterns themselves. Unlike the normal controls, a group of patients with unilateral surgical lesions that included the ANC (15 left, 18 right) did not show conditioned preferences, but performed normally on a measure of working memory. In contrast, 13 patients with unilateral damage confined to frontal cortex exhibited normal conditioned preferences but were impaired on the working memory task. This double dissociation provides clear evidence that, in humans as in other animals, reward-related learning (conditioned reward) critically depends on a circuit involving inferotemporal cortex and the ANC.

Key words: amygdaloid body; conditioning; emotion; frontal lobe; reward; temporal lobe; working memory

Everitt et al., 1991; Hiroi and White, 1991; White and McDonald, 1993) (for review, see Aggleton, 1993; McDonald and White, 1993; Gallagher and Holland, 1994; Ono et al., 1995; Hatfield et al., 1996; Gallagher and Chiba, 1996; Robbins and Everitt, 1996; Hitchcott et al., 1997; Hitchcott and Phillips, 1998; Everitt et al., 1999; Holland and Gallagher, 1999). Place-preference conditioning is one of the most common procedures for assessing conditioned reward associations (Carr et al., 1989; Schechter and Calcagnetti, 1993; Tzschentke, 1998). In this paradigm, a particular set of environmental cues is first paired with reward, and then an animal's tendency to approach and spend time in that environment, compared to a neutral one, is assessed. Placepreference conditioning procedures can be considered a special case of a more general conditioning paradigm (stimulus-reward association learning) in which biologically relevant stimuli (which normally elicit approach responses due to their rewarding affective significance) become associated with neutral stimuli that subsequently elicit approach on their own.

Conditioned preferences have been demonstrated previously in healthy human participants (Razran, 1954; Staats and Staats 1957, 1958; Levey and Martin, 1975, 1983; Martin and Levey, 1978; Kirk-Smith et al., 1983; Bierley et al., 1985; Rozin and Zellner, 1985; Stuart et al., 1987, 1991; Allen and Janiszewski, 1989; Niedenthal, 1990; Baeyens et al., 1993, 1995; Todrank et al., 1995), but the underlying anatomical substrates have not been examined in any detail. A few studies have investigated mechanisms of preference formation in brain-damaged populations, but these have focussed on the effects of exposure alone, rather than evaluating the effects of associative learning (Redington et al., 1984; Johnson et al., 1985). 
Table 1. Demographic information on participants in each group

\begin{tabular}{|c|c|c|c|c|c|c|c|c|c|c|c|c|c|}
\hline \multirow[b]{2}{*}{ Group } & \multirow[b]{2}{*}{$N$} & \multicolumn{2}{|c|}{ Sex } & \multicolumn{2}{|c|}{ Handedness } & \multicolumn{2}{|c|}{ Testing language } & \multicolumn{2}{|l|}{ Age } & \multicolumn{2}{|c|}{ Years of education } & \multicolumn{2}{|c|}{ Time since surgery } \\
\hline & & $\mathrm{M}$ & $\mathrm{F}$ & $\mathrm{R}$ & $\mathrm{L}$ & English & French & Mean & Range & Mean & Range & Median & Range \\
\hline LA & 15 & 12 & 3 & 15 & 0 & 13 & 2 & 36 & $23-48$ & 13 & $9-18$ & 4 years & 3 months -27 years \\
\hline RA & 18 & 7 & 11 & 15 & 3 & 8 & 10 & 38 & $22-55$ & 12 & $6-18$ & 1 year & 3 months -16 years \\
\hline FNTL & 13 & 7 & 6 & 9 & 4 & 10 & 3 & 30 & $16-39$ & 14 & $9-20$ & 1 year & 3 months -10 years \\
\hline $\mathrm{NC}$ & 21 & 10 & 11 & 20 & 1 & 15 & 6 & 37 & $23-58$ & 13 & $11-18$ & & \\
\hline
\end{tabular}

In a recent study (Johnsrude et al., 1999), conditioned preferences for novel, abstract, monochrome patterns were induced in young, normal volunteer participants using a method based on cue, or place, preference procedures originally developed for use with rats (Carr et al., 1989). Care was taken to minimize the subjects' awareness of the experimental contingencies and of the goal of the study by presenting the abstract patterns in the context of a cognitively demanding working memory task. Participants subsequently preferred the pattern paired most often with reward to that paired least often with reward. Importantly, participants did not attribute their preferences to the conditioning procedure, but instead attributed them to the physical characteristics of the patterns themselves, indicating that they were largely unaware of the effects of the conditioning procedure on their subsequent behavior.

In the present study, we used this procedure to assess preference conditioning in a group of neurosurgical patients with known damage to the amygdaloid region. In the majority of these patients, surgery was used to control pharmacologically intractable epilepsy. The resection always included the ANC and immediately surrounding tissue, as well as a variable amount of hippocampus, parahippocampal gyrus, and lateral neocortex in one hemisphere. The documented neuropsychological deficits after unilateral lesions of this type depend on the extent of the removal, but are generally mild and do not preclude the patient having a normal and productive life (Hermann et al., 1991; Eliashiv et al., 1997). We tested a matched group of normal volunteers and a group of patients with surgical lesions confined unilaterally to frontal cortex to evaluate the anatomical specificity of any resulting deficit.

\section{MATERIALS AND METHODS}

Participants. Thirty-three patients with unilateral anterior temporal lobe resections (15 left; 18 right) who had undergone surgery at the Montreal Neurological Institute for the relief of intractable epilepsy or for the removal of benign tumors were included in this study. In all of these patients, the lesion included the ANC and periamygdalar cortices, as described below. The study was approved by the Research and Ethics Committee of the Montreal Neurological Institute and Hospital, and all participants gave written consent. Patients with brain damage outside of the operated zone were excluded, and so were those who exhibited no improvement in their seizure frequency or intensity after surgery. All patients were either right-handed, or were left-hemisphere dominant for speech as determined by a preoperative sodium amobarbital test (Milner, 1997), and had full-scale intelligence quotient (IQ) ratings over 80 on the Wechsler Adult Intelligence Scale-Revised (WAIS-R).

Table 1 presents demographic information on participants in the four groups. The 15 patients with left ANC removals (LA) included one case of cortical dysplasia and one low-grade ganglioglioma. The pathology in the rest of the patients in this group was focal gliosis and sclerosis. Three of these patients were tested 3-4 months after surgery, two were tested 5 months to a year after surgery, three were tested between 1 and 5 years after surgery, and the rest were tested $>10$ years after surgery. Two of the 18 patients with removals of the right ANC (RA) were operated for neuroepithelial tumors, and one for a low-grade oligodendroglioma. The pathology in the rest of the group was gliosis and sclerosis. Seven of these patients were tested 3-4 months after surgery, two were tested 5 months to a year after surgery, two were tested between 1 and 5 years after surgery, another two were tested between 5 and 10 years after surgery, and the rest were tested more than 10 years after surgery. A group of 13 surgical patients with lesions confined unilaterally to frontal cortex (FNTL; seven patients with left-sided removals, six patients with rightsided removals) were also tested. Pathology reports cited low-grade gliomas in five cases (two left, three right) a cavernous hemangioma in one left-sided case, an aneurysm and hematoma in another left-sided patient, an arteriovenous malformation (AVM) in a right-sided patient, and cortical dysplasia, gliosis, and/or sclerosis in the other five patients. The resection did not encroach on temporal lobe structures in any of these patients. Two of these patients were tested 3-4 months postoperatively, five were tested 5 months to a year after surgery, three were tested between 1 and 5 years after surgery, two others were tested 5-10 years after surgery, and one was tested more than 10 years after surgery. Twenty-one neurologically normal volunteers (NC) who were matched to the patients with respect to age and years of education were also tested. One-way ANOVAs demonstrated no difference in age or years of education among the four groups. Nonparametric (Kruskal-Wallis) ANOVAs did not reveal any significant differences in the distributions of handedness, sex, or testing language (English or French) across the four groups.

Imaging in patients with temporal lobe resections. For conclusions about functional specialization within the medial temporal lobe to be drawn, it was important to confirm the site and extent of the resections in the patients with temporal lobe resections, and quantify the degree of overlap. The resections were labeled on postoperative T1-weighted magnetic resonance (MR) images, acquired on each subject (Philips Gyroscan; 1.5 $\mathrm{T})$. These images were linearly transformed into standardized stereotaxic space (Talairach and Tournoux, 1988), using DISPLAY, an interactive three-dimensional imaging software package (MacDonald et al., 1994; McConnell Brain Imaging Center, Montreal Neurological Institute). Postoperative MR images were not available for two patients in the LA group. The surgical reports for these two patients describe left anterior temporal lobe resections that include the amygdala and uncus, the pes of the hippocampus in one case, $3 \mathrm{~cm}$ of hippocampus in the other, and lateral neocortical resections in both extending to $\sim 4.5 \mathrm{~cm}$ along the Sylvian fissure and along the base of the temporal lobe, measured from the temporal pole. A postoperative MR image was not available for one patient in the RA group. The surgical report for this patient describes a right anterior temporal lobe resection including the amygdala and uncus, $3 \mathrm{~cm}$ of the hippocampus, and a lateral neocortical resection extending to $4 \mathrm{~cm}$ along the Sylvian fissure and $6 \mathrm{~cm}$ along the base of the temporal lobe. The label volumes resulting from analysis of the 13 postoperative MRs in the LA group and the $17 \mathrm{MRs}$ in the RA group were coregistered and averaged to produce probability volumes of lesion location, as shown in Figure 1. The area of maximal overlap in resection site $(100 \%$, resected in all patients) in each group is centered on the ANC (Talairach coordinates: LA, $-24,-15,-18$; RA, 24, -15, -19)

Materials and procedure. A computerized touch-screen task based on place-preference procedures used with rats was used. This task has been described in detail elsewhere (Johnsrude et al., 1999). Participants chose between raisins and fruit-flavored candy pellets (Dweebs; The Willy Wonka Candy Factory, Nestle, York, UK) before the start of the procedure, and they were always rewarded with that type of food. Raisins were chosen by 16 of 21 participants in the NC group, 12 of 15 patients in the LA group, 16 of 18 in the RA group, and 8 of 13 in the FNTL group. The rest chose candy pellets. These proportions are not significantly different across groups, as tested using $\chi^{2}$ tests. 


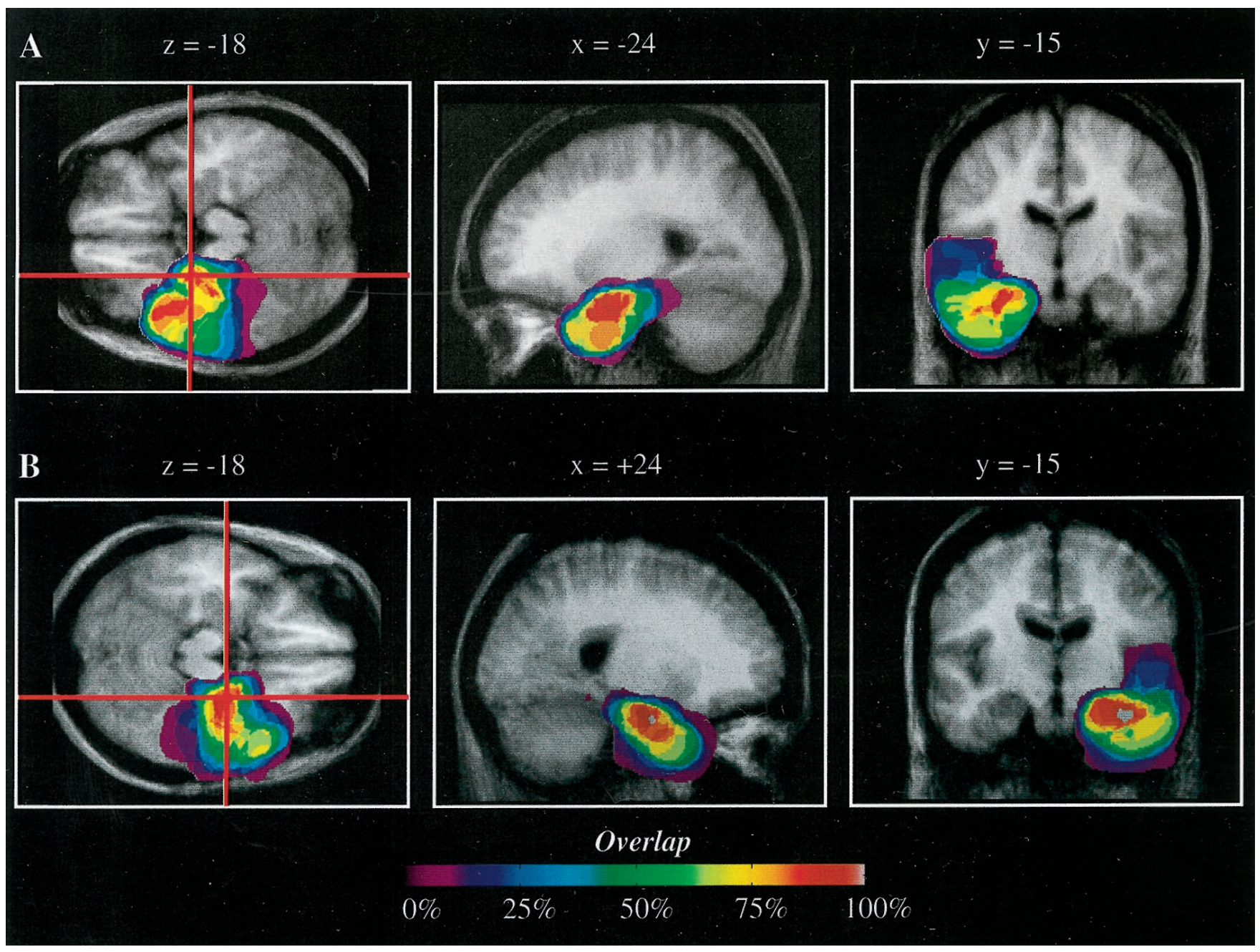

Figure 1. Overlap of lesions in patients with left (LA) or right (RA) anterior temporal lobe resection, as indexed by the color scale, superimposed on the averaged MR image for each group. $A, n=13$ LA patients. $B, n=17 \mathrm{RA}$ patients. In both LA and RA groups, the area of maximal overlap in lesion location includes the region of the amygdala.

There were three phases to the experimental procedure: formation of conditioned preferences, a test of preference expression, and an assessment of preference attribution. These are shown schematically in Fig. 2.

At the beginning of the formation phase, participants were presented with three black squares on the screen and were given the following instructions:

"You see three boxes on the screen. At any time, one of the boxes is hiding a red ball, and the other two are hiding black balls. What you have to do is guess where the red ball is. I would like you to find as many red balls as you can. You can choose a box by lightly touching the screen. Once you have touched a box, it will open up and show you which ball was hidden underneath. Every so often, you will be asked how many times you have found the red ball in a particular box. Thus, while you are choosing boxes you have also to try and remember how many times you have found a red ball in each of the three boxes. I would like you to eat one candy/raisin every time you find a red ball."

The participants then proceeded to guess where the red "ball" was hidden by touching one of the three black "boxes." After each guess, the selected box would "open" revealing one of three abstract black-andwhite line drawings or patterns, and either a red or a black circle (or ball) superimposed on the center of that pattern (Fig. $2 A$ ) If the circle was red, the participants heard a melodic flourish and picked the chosen type of food reward (one candy or one raisin) from a bowl placed beside the computer screen. If the circle was black, they heard a buzzer and were not permitted to take a food reward. After $3 \mathrm{sec}$, the selected box returned to black, and the subject was required to make the next guess. This interval ensured that participants had sufficient time to taste the food in the presence of the stimulus patterns on the rewarded trials.
Unknown to the subjects, the stimulus pattern and circle color were predetermined for each trial, regardless of the location chosen.

A total of 180 trials were presented over six blocks. In total, each of the stimulus patterns was presented 60 times, together with either a red ball or a black ball according to the contingency relationship for that pattern (see below). At the end of each block, the participants were asked how many times they had found the red circle in each of the three boxes during the previous block of trials; this was the working memory task.

Three versions of the formation procedure were prepared. Each subject was tested using one of the three different versions, chosen pseudorandomly, in such a way that the distribution of the versions across the sexes and across the reward types (candy or raisins) was approximately equal. In each version, a different set of pattern-outcome contingency pairings was used. Thus, in one version, pattern A was accompanied by reward (red circle, melodic flourish, and food reward) on $90 \%$ of trials in which it appeared (i.e., 54 trials) and by nonreward (black circle, buzzer sound and no food) on $10 \%$ of those trials (i.e., six trials). Pattern B was accompanied by reward on $50 \%$ of trials in which it appeared and by nonreward on the other $50 \%$. Pattern $\mathrm{C}$ was accompanied by reward on $10 \%$ of trials and by nonreward on the other $90 \%$. In the second version of the task the ratios were: Pattern A, 10:90; Pattern B, 90:10; Pattern C, 50:50, whereas in the third version, the corresponding ratios were: 50:50; 10:90, and 90:10.

The trial order was pseudorandom and fixed. The rarest combinations were always presented just before or just after the more frequent combinations (e.g., for the first version described above, Pattern A paired with a black circle was presented just after Pattern A paired with a red circle). In addition, an identical pattern/outcome pair could not occur 


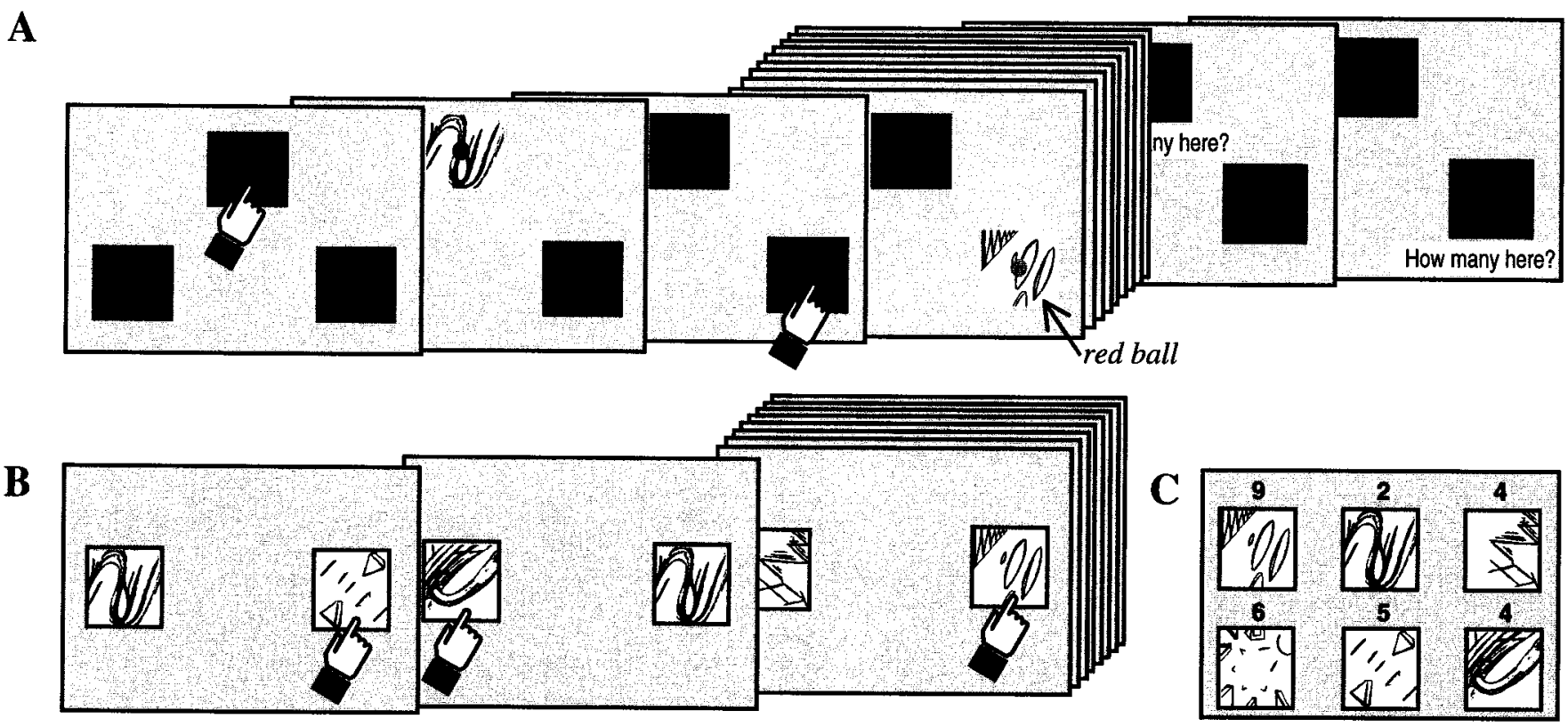

Figure 2. The three phases of the experimental procedure. A, Schematic drawing of a block of trials in the formation phase. On the first trial, the subject picked the top box and heard a buzzer at the same time as the pattern and black ball appeared. On the second trial, the subject selected the rightmost box and heard a melodic flourish at the same time as the pattern and the red ball appeared. After seeing the red ball, they took the chosen food reward (one candy or raisin) from the bowl beside the monitor. At the end of each block of trials the subject was asked to remember how many times they had found the red ball in each of the three boxes. $B$, Expression test. The subject saw successive pairs of patterns and was told to "touch the pattern you prefer." The six patterns used in this phase included the three used in the formation phase and three novel ones. $C$, In the final phase, the subject was shown the six patterns used in the preference expression test together with a number representing the number of times that pattern had been chosen (of 10). For the three patterns most frequently chosen, the subject was asked, "Why did you like this pattern?" to assess whether participants attributed their preferences to their experience during the formation phase.

more than twice in a row. These provisions served to break up runs of similar trials that might otherwise have alerted the participants to the different outcome contingencies. In addition, each block of trials contained an equal number of red and black circles, and at least one occurrence of each of the six possible combinations of circles and patterns.

Immediately after the conditioning procedure, preferences were assessed. Six different patterns were used in this part of the study: three of the patterns were those used in the conditioning procedure, whereas three others were novel. On each trial, a pair of patterns was presented, one on each side of the screen, as shown in Figure $2 B$. The participants were told to choose the pattern they preferred, by touching it. They were told not to think too hard about it, but to go with their first impression. There were a total of 30 trials, and each pattern was presented 10 times: five times on the left, and five times on the right, twice with each of the other five patterns.

After the preference assessment all six patterns were presented simultaneously on the screen for the preference attribution test. A number on top of each pattern indicated the number of times (out of 10) the subject had chosen each one (Fig. 2C). To assess the participants' perceptions of their preferences, they were asked, "Why did you like this pattern?" for their three most frequently chosen patterns. At the end of this phase, each participant was informed of the nature of the study.

Data scoring. The dependent variables analyzed included the preference scores for each pattern (the number of times, out of a possible ten, that it was chosen in the preference assessment phase), and counting error in the working memory component of the formation procedure. This variable was calculated by computing, for each of the boxes (left, top, and right) in each block of trials, the absolute discrepancy between the observed number of red balls (how may the subject actually saw) and the estimated number (how many the subject reported). These discrepancy values were summed across boxes and across blocks of trials to determine the overall discrepancy score. The discrepancy values were also categorized as underestimations or overestimations, and the values were summed separately to determine the total amount of each type of error for each subject. One LA subject had a total discrepancy score $>3$ SDs away from the mean for this group: his data were not included in the analysis.

\section{RESULTS}

It was decided, a priori, to exclude from the analysis data from those participants who demonstrated any spontaneous knowledge of the relationship between their experience with the patterns and their preferences. In fact, none of the participants related their preferences to the previous stage of the task during the preference attribution test or debriefing. Instead, they attributed their preferences to the physical characteristics of the patterns themselves. Sample responses (from normal participants and patients with frontal lobe damage) to the question, "Why did you like this pattern?" included "looks like the sun", "reminds me of pizza", "has more of a design, is a little more complicated", "is symmetrical", "I liked the lines and curves", and "was an interesting sort of pattern, and caught my eye."

When the preference scores for the 90 and $10 \%$ patterns were compared across groups using a two-way ANOVA, a significant group-by-pattern interaction was obtained, $\left(F_{(3,63)}=4.09 ; p<\right.$ $0.01)$. Planned comparisons between the two preference scores in each group demonstrated that participants in the NC and FNTL groups preferred the $90 \%$ pattern to the $10 \%$ pattern $\left(\mathrm{NC}: t_{(20)}=\right.$ $4.0, p<0.0005$; FNTL: $t_{(12)}=1.92, p<0.05$, one-tailed), whereas LA and RA participants did not (Fig. $3 A$ ). The LA group tended to prefer the $10 \%$ pattern to the $90 \%$ pattern, but not to a significant degree, $t_{(14)}=1.35, p=0.1$.

Preference scores for the $50 \%$ pattern and for the novel patterns were intermediate between those for the 10 and the $90 \%$ patterns (range of mean preference scores on $50 \%$ pattern, 3.95.7; novel patterns, 4.9-5.4). These patterns were included for reasons of experimental design, and we did not expect or observe any differences among the groups in preference scores for these patterns (as tested using one-way ANOVAs). Furthermore, the 
A

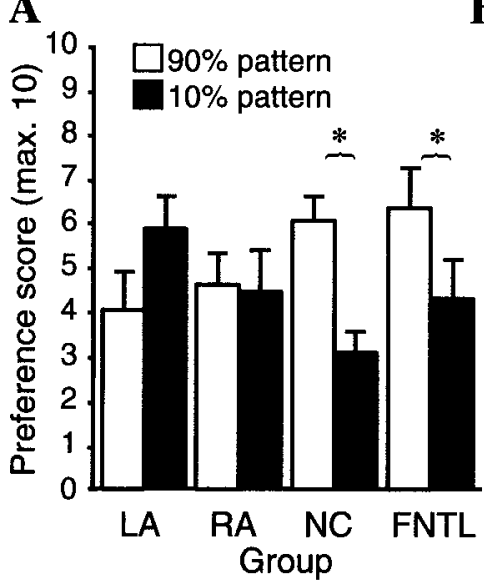

B

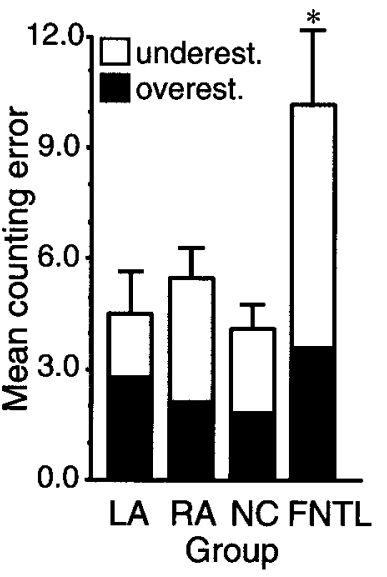

Figure 3. A, The mean preference scores (maximum $=10$ ) and SEs for the 90 and $10 \%$ patterns in patients with left or right temporal lobe damage, including the amygdaloid body ( $L A, R A)$, normal control participants $(N C)$, and patients with unilateral frontal lobe lesions $(F N T L)$. Error bars indicate SEM. LA and RA participants fail to show a preference effect; see Results. $B$, Mean discrepancy scores on the working memory task in normal control participants $(N C)$ and in patients with left $(L A)$ or right $(R A)$ anterior temporal lobe resection and frontal lobe resections $(F N T L)$. The patients with frontal lobe lesions are impaired relative to all other groups. Error bars indicate SEM for the overall discrepancy scores. When the errors are categorized as overestimations or underestimations, the FNTL group is shown to produce significantly more underestimations relative to the other three groups; see Results.

value 5, which is the value expected if participants were choosing at random during the preference assessment phase, was within 1 $\mathrm{SD}$ of the cell mean preference scores for these patterns.

One-way ANOVA on the overall discrepancy scores revealed that NC, LA, and RA participants were equally able to keep track of the number of times they had found the red ball in each of the three boxes, whereas patients with frontal lobe damage were significantly impaired [Tukey's honestly significant difference (HSD) tests: main effect, $\left.F_{(3,62)}=5.22 ; p<0.005\right]$. When the discrepancy values were categorized as overestimations and underestimations, it was apparent that patients with frontal lobe damage significantly underestimated the number of times they had found the red ball in each location (Tukey's HSD tests after one-way ANOVA: $\left.F_{(3,62)}=7.92 ; p<0.0005\right)$. There were no differences among the groups in overestimation (one-way ANOVA: $F_{(3,62)}=1.35 ; p$, ns). This is shown in Figure $3 B$. Total discrepancy, overestimation, and underestimation scores for patients with left frontal $(n=7)$ and right frontal $(n=6)$ excisions were then examined separately to look for hemisphere effects. Mann-Whitney $U$ tests were used to compare these two subgroups with normal participants and with each other. Both frontal groups differed significantly from normals on total discrepancy scores and underestimation scores ( $p$ values $<0.05$ ), but not on overestimation scores, consistent with the $F$ tests. Left frontal and right frontal groups did not differ on any measure.

\section{DISCUSSION}

In a previous experiment, a task based on place-preference conditioning procedures was used to induce conditioned reward associations in normal human volunteers (Johnsrude et al., 1999). The same task was used in the present study to investigate whether the ANC might be critically involved in conditioned reward learning in humans, as it is in other animals. Compared to normal control subjects and patients with unilateral frontal cortex excisions, patients with unilateral anterior temporal lobe resections that included the amygdaloid complex showed severely impaired preference conditioning. In contrast, patients with anterior temporal lobe resections performed normally on the counting task, whereas patients with frontal cortex damage were severely impaired. This task, which required that subjects monitor the frequency of occurrence of red balls in each of the three boxes, places significant demands on aspects of working memory that are known to depend on the integrity of the prefrontal cortex rather than the medial temporal lobe structures (Petrides and Milner, 1982; Smith and Milner, 1988; Owen et al., 1990, 1996; Jonides et al., 1993; Smith, 1996; Owen, 1997). This double dissociation provides clear evidence that, in humans as in other animals, reward-related learning (conditioned reward) critically depends on a circuit involving inferotemporal cortex and the ANC.

Several features of the experimental design were included to minimize the participants' awareness of the conditioning procedure and the goals of the experiment. The subjects' attention was not explicitly drawn to the stimuli to be conditioned before or during conditioning, and these stimuli were irrelevant to performance of the counting task. Furthermore, the counting task was made quite demanding to minimize the subjects' opportunity to attend to anything but that task and the occurrence of reward or nonreward. The three patterns were paired with reward on 90, 50, and $10 \%$ of the trials in which they occurred. By avoiding the absolutes of 100 and $0 \%$, and by including a pattern paired on equal number of trials with reward and nonreward, the experimental contingencies were made more difficult to discern. Participants were also asked, at the end of the study, why they showed the preferences they did, to determine whether preferences would be attributed to previous experience with the patterns. Without exception, participants attributed their preferences to the characteristics of the pattern itself. Because the patterns were randomly assigned to the different reward contingencies for each subject, these responses may be subjective rationalizations of a conditioned preference. This suggests that participants were largely unaware of the effect of their previous experience on their behavior. The patients with temporal lobe removals also attributed their preferences to the characteristics of the patterns themselves. They did show preferences, but their preferences were not consistently related to the frequency with which patterns were associated with reward in the conditioning procedure. They did not "learn to like", as the normal subjects and those with frontal lobe damage did.

All patients in the LA and RA groups had extensive damage to the amygdaloid region, including the perirhinal and entorhinal cortices, as well as white matter tracts passing through this area (Fig. 1). Both groups, particularly the LA group, also had significant damage to anterior temporal neocortex. Work in monkeys indicates that bilateral lesions of either the anterior part of inferior temporal cortex or the ANC (but not the posterior part of inferior temporal cortex or the hippocampus) produce impairments in object-reward association learning (Jones and Mishkin, 1972; Speigler and Mishkin, 1981; Gaffan and Harrison, 1987). The impairments seen in many of these studies may not be attributable to damage to the ANC per se, but may be caused by entorhinal and/or perirhinal cortex damage, or to damage to white matter tracts passing through the amygdalar region that normally connect rhinal and more lateral temporal cortex with ventral frontal cortex and medial thalamus (see, for example, Málková et al., 1997). However, considerable evidence from ex- 
periments with rats does implicate the ANC, particularly the lateral and basolateral nuclei to the exclusion of other surrounding tissue, in conditioned reward (Peinado-Manzano, 1987, 1988; Cador et al., 1989; Everitt et al., 1989, 1991; Everitt, 1990; McDonald and White, 1993; White and Hiroi, 1993). The lesions in the patients we tested encompassed the amygdala and surrounding tissue and so we are unable to draw conclusions about functional specialization of structures within this region. A notable difference between the present study and the existing animal literature is that the amygdala is typically lesioned or inactivated bilaterally in animal studies of reward-related learning (ColemanMesches et al., 1996). The LA and RA participants tested in the present study had predominantly unilateral damage, although undiagnosed dysfunction in some structures contralateral to the site of the excised epileptic focus may have contributed to the impairment (Incisa della Rocchetta et al., 1995). LaBar et al. (1995) found significant impairments in fear conditioning in unilateral temporal lobectomy patients, also with no effect of side of excision.

The findings presented here of impaired preference conditioning in patients with even unilateral lesions of the medial temporal area contrast with those of Tranel and Damasio (1993), who found evidence for preserved learning of affective associations in Boswell, a patient with bilateral medial-temporal damage (including the ANC). In that study, three previously novel people were instructed to act toward Boswell in either a positive (e.g., giving compliments and treats), negative (e.g., requiring him to participate in tedious experiments), or neutral way, over a $5 \mathrm{~d}$ period. Boswell was subsequently unable to identify the stimulus persons, but when asked to "choose the person that you would go to for a treat" he consistently chose the positive stimulus person, suggesting that he was able to learn to associate faces with reward outcomes. However, Tranel and Damasio (1993) speculate that Boswell's preserved learning might reflect the action of another learning system, that for reinforced stimulus-response associations. This type of learning appears to be mediated by a neural system that includes the dorsal striatum (e.g., Mishkin et al., 1985; McDonald and White, 1993), which is intact in Boswell. This learning system would also be expected to be intact in our patients with more restricted lesions. The apparent discrepancy in the results of the two studies therefore probably reflects the different requirements of the two tasks.

Because the anterior temporal lobe is considered to be the final stage in the cortical visual system, our results accord well with the idea that visual stimuli acquire affective significance through an interaction between high-level visual areas and the ANC. Temporal lobe excisions do not generally result in marked visual perceptual impairments, although patients with right temporal lobe lesions can show subtle deficits when normal stimulus redundancy has been reduced, by presenting items tachistoscopically, for example (Milner, 1990). The stimuli used in the present study were not degraded in any way, and all patients in both temporal lobe groups appeared able to discriminate them, judging from their physical descriptions of the patterns during the last phase.

The findings of the present study are also consistent with a body of literature demonstrating that aspects of working memory may be dependent on the integrity of the prefrontal cortex (Petrides and Milner, 1982; Funahashi et al., 1989; Owen et al., 1990, 1996; Jonides et al., 1993; Petrides et al., 1993; Wilson et al., 1993; Cohen et al., 1994; Courtney et al., 1996; Rao et al., 1997) (for review, see Owen, 1997; Rushworth and Owen, 1998). Thus, unlike the anterior temporal lobe group, the patients with exci- sions of the frontal lobe were unable to maintain an ongoing record of how many red balls had been found in each location, a requirement that undoubtedly placed demands on aspects of working memory.

Smith and Milner (1988) and Smith (1996) presented series of words and abstract designs to normal volunteers and patients with unilateral frontal or temporal lobe excisions. The words and designs differed in the number of times that they were repeated within a series. Whereas normal participants and patients with anterior temporal lobe resections were equally able to estimate frequency of occurrence, patients with frontal lobe excisions, particularly the right frontal group, significantly underestimated the frequency of occurrence of either words or designs. Furthermore, Smith noticed a trend toward material-specificity in this deficit: patients with left frontal-lobe lesions were more impaired than patients with right frontal lobe lesions at estimating the frequency of occurrence of words, and the reverse pattern was observed for designs. No effect of side of excision was seen in the present study: patients with right and left frontal lobe resections appeared equally impaired. The lack of hemispheric asymmetry may be owing to the nature of the stimuli to be monitored in the present study: participants were required to monitor the conjunction of a particular event (red ball) with three distinct locations (left, top, or right). This is qualitatively different from monitoring the occurrence of words or abstract designs that are all presented in the same spatial position.

In conclusion, the results of this study suggest that working memory and stimulus-reward association learning are doubly dissociable in humans. Patients with unilateral frontal lesions were impaired at the working memory component of the task, but did show preference conditioning. Conversely, patients with anterior temporal lobe resections performed normally on the working memory component, but did not acquire stimulus-reward associations. To date, the work on mediation of emotional learning by the ANC in humans has focussed on negative affect, such as fear (Bechara et al., 1995; LaBar et al., 1995, 1998; Büchel et al., 1998; Morris et al., 1998; but see, Hamann et al., 1999). The results presented in this paper demonstrate that, in humans as in other species, structures in the region of the amygdala are required for cues to acquire affective significance through their association with rewarding events.

\section{REFERENCES}

Aggleton JP (1993) The contribution of the amygdala to normal and abnormal emotional states. Trends Neurosci 16:328-333.

Allen CT, Janiszewski CA (1989) Assessing the role of contingency awareness in attitudinal conditioning with implications for advertising research. J Marketing Res 26:30-43.

Baeyens F, Hermans D, Eelen P (1993) The role of CS-US contingency in human evaluative conditioning. Behav Res Ther 31:731-737.

Baeyens F, Eelen P, Crombez G (1995) Pavlovian associations are forever: on classical conditioning and extinction. J Psychophysiol 9:127-141.

Bechara A, Tranel D, Damasio H, Adolphs R, Rockland C, Damasio AR (1995) Double dissociation of conditioning and declarative knowledge relative to the amygdala and hippocampus in humans. Science 269:1115-1118.

Bierley C, McSweeney FK, Vannieuwkerk R (1985) Classical conditioning of preferences for stimuli. J Consumer Res 12:316-323.

Büchel C, Morris J, Dolan RJ, Friston KJ (1998) Brain systems mediating aversive conditioning: An event-related fMRI study. Neuron 20:947-957.

Cador M, Robbins TW, Everitt BJ (1989) Involvement of the amygdala in stimulus-reward associations: interaction with the ventral striatum. Neuroscience 30:77-86.

Carr GD, Fibiger HC, Phillips AG (1989) Conditioned place preference 
as a measure of drug reward. In: The neuropharmacological basis of reward (Leibman JM, Cooper SJ, eds), pp 264-319. Oxford: Oxford UP.

Cohen JD, Forman SD, Braver TS, Casey BJ, Servan-Schreiber D, Noll DC (1994) Activation of prefrontal cortex in a non-spatial working memory task with functional MRI. Hum Brain Mapp 1:293-304.

Coleman-Mesches K, Salinas JA, McGaugh JL (1996) Unilateral amygdala inactivation after training attenuates memory for reduced reward. Behav Brain Res 77:175-180.

Courtney SM, Ungerlieder LG, Keil K, Haxby JV (1996) Object and spatial visual working memory activate separate neural systems in human cortex. Cereb Cortex 6:39-49.

Davis M (1998) Anatomic and physiologic substrates of emotion in an animal model. J Clin Neurophysiol 15:378-387.

Eliashiv SD, Dewar S, Wainwright I, Engel J Jr, Fried I (1997) Longterm follow-up after temporal lobe resection for lesions associated with chronic seizures. Neurology 48:1383-1388.

Everitt BJ (1990) Sexual motivation: A neural and behavioural analysis of the mechanisms underlying appetitive and copulatory responses of male rats. Neurosci Biobehav Rev 14:217-232.

Everitt BJ, Cador M, Robbins TW (1989) Interactions between the amygdala and ventral striatum in stimulus-reward associations: studies using a second-order schedule of sexual reinforcement. Neuroscience 30:63-75.

Everitt BJ, Morris KA, O’Brien A, Robbins TW (1991) The basolateral amygdala-ventral striatal system and conditioned place preference: further evidence of limbic-striatal interactions underlying rewardrelated processes. Neuroscience 42:1-18.

Everitt BJ, Parkinson JA, Olmstead MC, Arroyo M, Robledo P, Robbins TW (1999) Associative processes in addition and reward. The role of amygdala-ventral striatal subsystems. Ann N Y Acad Sci 877:412-438.

Fanselow M, LeDoux J (1999) Why we think plasticity underlying Pavlovian fear conditioning occurs in the basolateral amygdala. Neuron 23:229-232.

Fendt M, Fanselow MS (1999) The neuranatomical and neurochemical basis of conditioned fear. Neurosci Biobehav Rev 23:743-760.

Funahashi S, Bruce CJ, Goldman-Rakic PS (1989) Mnemonic coding of visual space in the monkey's dorsolateral prefrontal cortex. J Neurophysiol 61:1-19.

Gaffan D, Harrison S (1987) Amygdalectomy and disconnection in visual learning for auditory secondary reinforcement by monkeys. J Neurosci 7:2285-2292.

Gallagher M, Chiba AA (1996) The amygdala and emotion. Curr Opin Neurobiol 6:221-227.

Gallagher M, Holland PC (1994) The amygdala complex: multiple roles in associative learning. Proc Natl Acad Sci USA 91:11771-11776.

Gallagher M, Graham PW, Holland PC (1990) The amygdala central nucleus and appetitive Pavlovian conditioning: lesions impair one class of conditioned behavior. J Neurosci 10:1906-1911.

Hamann SB, Ely TD, Grafton ST, Kilts CD (1999) Amygdala activity related to enhanced memory for pleasant and aversive stimuli. Nat Neurosci 2:289-294.

Hatfield T, Han J-S, Conley M, Gallagher M, Holland P (1996) Neurotoxic lesions of basolateral, but not central, amygdala interfere with Pavlovian second-order conditioning and reinforcer devaluation effects. J Neurosci 16:5256-5265.

Hermann BP, Wyler AR, Somes G (1991) Preoperative psychological adjustment and surgical outcome are determinants of psychosocial status after anterior temporal lobectomy. J Neurol Neurosurg Psychiatry 55:491-496.

Hiroi N, White NM (1991) The lateral nucleus of the amygdala mediates expression of the amphetamine-produced conditioned place preference. J Neurosci 11:2107-2116.

Hitchcott PK, Phillips GD (1998) Double dissociation of the behavioural effects of $\mathrm{R}(+)$ 7-OH-DPAT infusions in the central and basolateral amygdala nuclei upon Pavlovian and instrumental conditioned appetitive behaviours. Psychopharmacology 140:458-469.

Hitchcott PK, Bonardi CM, Phillips GD (1997) Enhanced stimulusreward learning by intra-amygdala administration of a D3 dopamine receptor agonist. Psychopharmacology 133:240-248.

Holland PC, Gallagher M (1999) Amygdala circuitry in attention and representational processes. Trends Cognit Sci 3:65-73.

Incisa della Rocchetta A, Gadian DG, Connelly A, Polkey CE, Jackson GD, Watkins KE, Johnson CL Mishkin M, Vargha-Khadem F (1995) Verbal memory impairment after right temporal lobe surgery: role of contralateral damage as revealed by $1 \mathrm{H}$ magnetic resonance spectroscopy and T2 relaxometry. Neurology 45:797-802.

Johnson MK, Kim JK, Risse G (1985) Do alcoholic Korsakoff's syndrome patients acquire affective reactions? J Exp Psychol: Learn Mem Cog 11:22-36.

Johnsrude IS, Owen AM, Zhao WV, White NM (1999) Conditioned preference in humans: a novel experimental approach. Learn Motiv 30:250-264.

Jones B, Mishkin M (1972) Limbic lesions and the problem of stimulusreinforcement associations. Exp Neurol 36:362-377.

Jonides J, Smith EE, Koeppe RA, Awh E, Minoshima S, Mintun MA (1993) Spatial working memory in humans as revealed by PET. Nature 363:623-625.

Killcross S, Robbins TW, Everitt BJ (1997) Different types of fearconditioned behaviour mediated by separate nuclei within the amygdala. Nature 388:377-380.

Kirk-Smith MD, van Toller C, Dodd GH (1983) Unconscious odour conditioning in human subjects. Biol Psychol 17:221-231.

LaBar KS, LeDoux JE, Spencer DO, Phelps EA (1995) Impaired fear conditioning following unilateral temporal lobectomy in humans. J Neurosci 15:6846-6855.

LaBar KS, Gatenby JC, Gore JC, LeDoux JE, Phelps EA (1998) Human amygdala activation during conditioned fear acquisition and extinction: A mixed-trial fMRI study. Neuron 20:937-945.

LeDoux JE (1998) Fear and the brain: where have we been, and where are we going? Biol Psychiatry 44:1229-1238.

Levey AB, Martin I (1975) Classical conditioning of human "evaluative" responses. Behav Res Ther 13:221-226.

Levey AB, Martin I (1983) Part I. Cognitions, evaluations and conditioning: rules of sequence and rules of consequence. Adv Behav Res Ther 4:181-195.

MacDonald JD, Avis D, Evans AC (1994) Multiple surface identification and matching in magnetic resonance images. Proc Soc Vis Biomed Comput 2359:160-169.

Málková L, Gaffan D, Murray EA (1997) Excitotoxic lesions of the amygdala fail to produce impairment in visual learning for auditory secondary reinforcement but interfere with reinforcer devaluation effects in rhesus monkeys. J Neurosci 17:6011-6020.

Martin I, Levey AB (1978) Evaluative conditioning. Adv Behav Res Ther 1:57-102.

McDonald RJ, White NM (1993) A triple dissociation of memory systems: hippocampus, amygdala, and dorsal striatum. Behav Neurosci 107:3-22.

Milner B (1990) Right temporal-lobe contribution to visual perception and visual memory. In: Vision, temporal lobe and memory (Iwai E, ed), pp 43-53. New York: Elsevier.

Milner B (1997) Amobarbital memory testing: Some personal reflections. Brain Cogn 33:14-17.

Mishkin M, Malamut B, Bachevalier J (1985) Memories and habits: two neural systems. In: Neurobiology of learning and memory (Lynch G, McGaugh JL, Weinberger NM, eds) New York: Guilford.

Morris JS, Öhman A, Dolan RJ (1998) Conscious and unconscious emotional learning in the human amygdala. Nature 393:467-470.

Niedenthal PM (1990) Implicit perception of affective information. J Exp Social Psychol 26:505-527.

Ono T, Nishijo H, Uwano T (1995) Amygdala role in conditioned associative learning. Prog Neurobiol 46:401-422.

Owen AM (1997) The functional organization of working memory processes within human lateral frontal cortex: the contribution of functional neuroimaging. Eur J Neurosci 9:1329-1339.

Owen AM, Downes JJ, Sahakian BJ, Polkey CE, Robbins TW (1990) Planning and spatial working memory following frontal lobe lesions in man. Neuropsychologia 28:1021-1034.

Owen AM, Morris RG, Sahakian BJ, Polkey CE, Robbins TW (1996) Double dissociations of memory and executive functions in working memory tasks following frontal lobe excisions, temporal lobe excisions or amygdalo-hippocampectomy in man. Brain 119:1597-1615.

Peinado-Manzano MA (1987) Role of the lateral nucleus of the amygdala in successive conditional visual discrimination learning in rats. Med Sci Res 15:383-384.

Peinado-Manzano MA (1988) Effects of bilateral lesions of the central and lateral amygdala on free operant successive discrimination. Behav Brain Res 29:61-72.

Petrides M, Milner BA (1982) Deficits on subject-ordered tasks after 
frontal- and temporal-lobe lesions in man. Neuropsychologia 20:249-262.

Petrides M, Alivisatos B, Evans AC, Meyer E (1993) Dissociation of human mid-dorsolateral from posterior dorsolateral frontal cortex in memory processing. Proc Natl Acad Sci USA 90:873-877.

Rao SC, Rainer G, Miller EK (1997) Integration of what and where in the primate prefrontal cortex. Science 276:821-824.

Razran GHS (1954) The conditioned evocation of attitudes (cognitive conditioning?) J Exp Psychol 48:278-282.

Redington K, Volpe BT, Gazzaniga MS (1984) Failure of preference formation in amnesia. Neurology 34:536-538.

Robbins TW, Everitt BJ (1996) Neurobehavioural mechanisms of reward and motivation. Curr Opin Neurobiol 6:228-236.

Robbins TW, Cador M, Taylor JR, Everitt BJ (1989) Limbic-striatal interactions in reward-related processes. Neurosci Biobehav Rev 13:155-162.

Rogan MT, Staubli UV, LeDoux JE (1997) Fear conditioning induces associative long-term potentiation in the amygdala. Nature 390:604-607.

Rozin P, Zellner D (1985) The role of Pavlovian conditioning in the acquisition of food likes and dislikes. In: Experimental assessments and clinical applications of conditioned food aversions (Braveman NS, Bronstein P, eds), pp 189-202. New York: N Y Acad Sci.

Rushworth MFS, Owen AM (1998) The functional organization of the lateral frontal cortex: conjecture or conjuncture in the electrophysiology literature. Trends Cognit Sci 2:46-53.

Schechter MD, Calcagnetti DJ (1993) Trends in place preference conditioning with a cross-indexed bibliography: 1957-1991. Neurosci Biobehav Rev 17:21-41.

Smith ML (1996) Recall of frequency of occurrence of self-generated and examiner-provided words after frontal or temporal lobectomy. Neuropsychologia 34:553-563.

Smith ML, Milner B (1988) Estimation of frequency of occurrence of abstract designs after frontal or temporal lobectomy. Neuropsychologia 26:297-306.

Speigler BJ, Mishkin M (1981) Evidence for the sequential participation of inferior temporal cortex and amygdala in the acquisition of stimulusreward associations. Behav Brain Res 3:303-317.
Staats AW, Staats CK (1958) Attitudes established by classical conditioning. J Abnorm Psychol 57:37-40.

Staats CK, Staats AW (1957) Meaning established by classical conditioning. J Exp Psychol 54:74-80.

Stuart EW, Shimp TA, Engle RW (1987) Classical conditioning of consumer attitudes: four experiments in an advertising context. J Consumer Res 14:334-349.

Stuart EW, Shimp TA, Engle RW (1991) A program of classical conditioning experiments testing variations in the conditioned stimulus and context. J Consumer Res 18:1-12.

Talairach J, Tournoux P (1988) Co-planar stereotaxic atlas of the human brain: 3-dimensional proportional system: an approach to cerebral imaging. Stuttgart, Germany: Thieme.

Todrank J, Byrnes D, Wrzesniewski A, Rozin P (1995) Odors can change preferences for people in photographs: a cross-modal evaluative conditioning study with olfactory USs and visual CSs. Learn Motiv 26:116-140.

Tranel D, Damasio AR (1993) The covert learning of affective valence does not require structures in hippocampal system or amygdala. J Cogn Neurosci 5:79-88.

Tzschentke TM (1998) Measuring reward with the conditioned pace preference paradigm: a comprehensive review of drug effects, recent progress and new issues. Prog Neurobiol 56:613-672.

Walker DL, Davis M (1997) Double dissociation between the involvement of the bed nucleus of the stria terminalis and the central nucleus of the amygdala in startle increases produced by conditioned versus unconditioned fear. J Neurosci 17:9375-9383.

White NM, Hiroi N (1993) Amphetamine conditioned cue preference and the neurobiology of drug seeking. Semin Neurosci 5:329-336.

White NM, McDonald RJ (1993) Acquisition of a spatial conditioned place preference is impaired by amygdala lesions and improved by fornix lesions. Behav Brain Res 55:269-281.

Wilson FA, O Scalaidhe SP, Goldman-Rakic PS (1993) Dissociations of object and spatial processing domains in primate prefrontal cortex. Science 260:1955-1958.

Weiskrantz L (1956) Behavioural changes associated with ablation of the amygdaloid complex in monkeys. J Comp Physiol Psychol 9: 381-391. 